\title{
A Synthetic Mimic of Inhibitor Binding in Phosphodiesterase Type 5 based on Corona Phase Molecular Recognition of Single Walled Carbon Nanotubes
}

\author{
Juyao Dong, Michael A. Lee, Imon Rahaman, Jessica H. Sun, Ananth Govind \\ Rajan, Daniel P. Salem, and Michael S. Strano* \\ Department of Chemical Engineering, Massachusetts Institute of Technology, \\ 77 Massachusetts Avenue, Cambridge, Massachusetts 02139 \\ E-mail: strano@mit.edu
}

\begin{abstract}
Molecular recognition binding sites that specifically identify a target molecule are essential for research in the life sciences, clinical diagnoses and therapeutic development. Corona Phase Molecular Recognition is a technique introduced to generate synthetic recognition at the surface of a nanoparticle corona, but it remains an important question whether such entities can achieve the specificity of natural enzymes and receptors. In this work, we generate and screen a library of 24 amphiphilic polymers based on functional monomers including methacrylic acid, acrylic acid, styrene and so on, iterating upon a poly(methacrylic acid-co-styrene) motif that demonstrates a binding specificity remarkably similar to an enzyme - Phosphodiesterase Type 5 - in its molecular recognition. The corona phase binds selectively to an inhibitor Vardenafil, as well as its derivatives, but not to another inhibitor and substrate that
\end{abstract}


interact differently with the enzyme. Our study examines the specificity and sensitivity by mutation of the polymer structure and configuration, as well as the competition with native binding sites. We demonstrate that the recognition originates from the unique three-dimensional configuration of the corona phase. This work conclusively shows that corona phase molecular recognition can mimic key aspects of biological recognition sites and drug targets, opening up possibilities for new pharmaceutical and biological applications.

\section{Introduction}

Molecular recognition sites are molecules that specifically bind to one target molecule with high affinity. They are indispensable 1) for biological research in studying proteins' correlation and function in signal cascades, 2) for clinical diagnosis in identifying trace amount of disease biomarkers in biofluids, and 3) for therapeutic development as they target abnormal bioactivities. However, the most commonly used molecular recognition sites - antibodies are produced by immune cells and have intrinsic large molecular weight. As a result, they suffer from low thermal stability, poor tissue penetration, immunogenicity, and high production cost. ${ }^{1-4}$ To solve this problem, extensive efforts have been focused on developing synthetic analogues to achieve similar recognition function.

Creating artificial recognition sites, however, is a grand challenge. Early investigations modified macrocyclic molecules, such as cucurbituril and crown ether, using their hydrophobic interior to interact with target small molecules, like dopamine, with limited selectivity. ${ }^{5,6}$ Later, molecular imprinting was invented to carry out polymerization in the presence of analyte as the template, so that after extracting the template, the resulting polymer conformation has a cavity with a favorable shape and functional residues. The method, however, is limited by monomer options, polymerization conditions, incomplete template removal, and low binding capacity. ${ }^{7,8}$ In addition to synthetic methods, combinatorial screening of macromolecules is the main strategy in generating recognition sites for large biomolecules. ${ }^{9-12}$ For 
example, random single stranded DNAs are screened against a target protein, where potential binding sequences are enriched to generate DNA aptamers. ${ }^{11}$ Similar methods using other sequence-defined polymers, like peptides and non-natural foldamers have also been developed. ${ }^{10,12}$ This combinatorial screening strategy is very time-consuming and resource demanding, requiring a large structure library (usually on the orders of thousands or millions of molecules), complex identification procedures, and multiple rounds of optimization.

To rationally design artificial recognition sites, our strategy is to introduce a rigid nano structure as a scaffold to direct the folding of linear molecules, therefore to manipulate the 3D configurations. More specifically, we use single-walled carbon nanotubes (SWNTs) - a high aspect ratio nanostructure - to guide polymer folding through supramolecular interactions. The resulting polymer corona together with the nanotube surface produces a unique configuration for recognizing target molecules.

In addition to the structural function, SWNTs also serve as signal transducer in identifying recognition interactions. Semiconductor SWNTs have near infrared (nIR) fluorescence. Because all atoms in nanotubes are surface atoms, the fluorescence is very sensitive to local dielectric environment change. A recognition interaction with the target analyte can modulate the effective dielectric constant or provide extra relaxation modes, both of which will change nanotubes' nIR emission, serving as a direct macroscopic readout of the molecular-level interactions. Therefore, our approach combines molecular recognition and signal transduction into one platform. The strategy has been applied to analytes including nitric oxide, hydrogen peroxide, dopamine and fibrinogen, ${ }^{13-16}$ but has not been examined for its specificity in comparison with the natural binding site.

In this work, we present the creation of an artificial molecular recognition site that mimics the interaction between enzyme phosphodiesterase type 5 (PDE5) and its inhibitor Vardenafil. A library of 24 amphiphilic polymers are templated to fold on nanotube surfaces, and one resulting corona phase selectively interacts with Vardenafil. For this interaction, we examine its specificity, its sensitivity to corona configuration and analyte inhibitors, and its 
comparison and competition with natural enzyme. Together with the simulation results, we show that our corona phase imitates PDE5 enzyme in its interaction with Vardenafil, which is responsible for the specific recognition. We demonstrate that corona phase molecular recognition can mimic key aspects of biological recognition sites and provide new strategy for pharmaceutical applications.
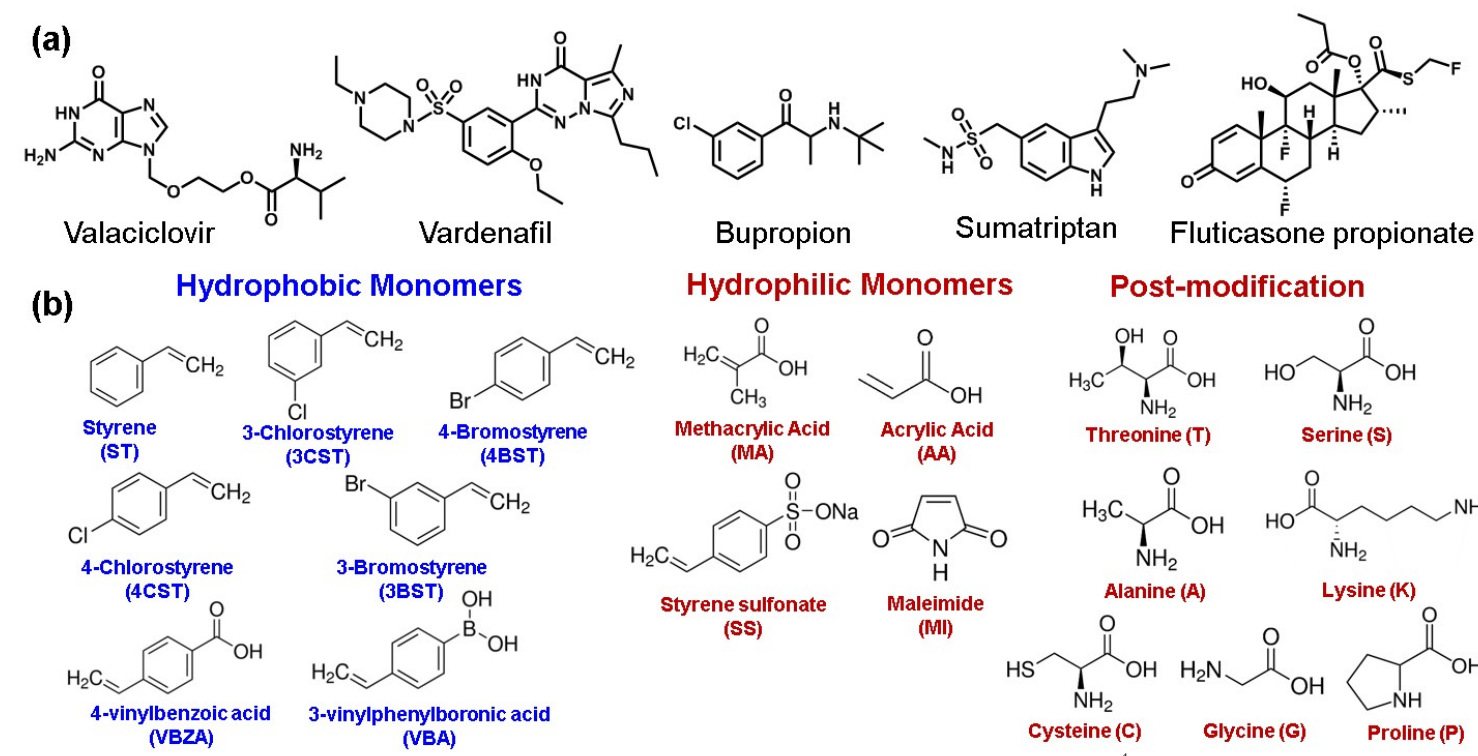
Hydrophilic Monomers Post-modification
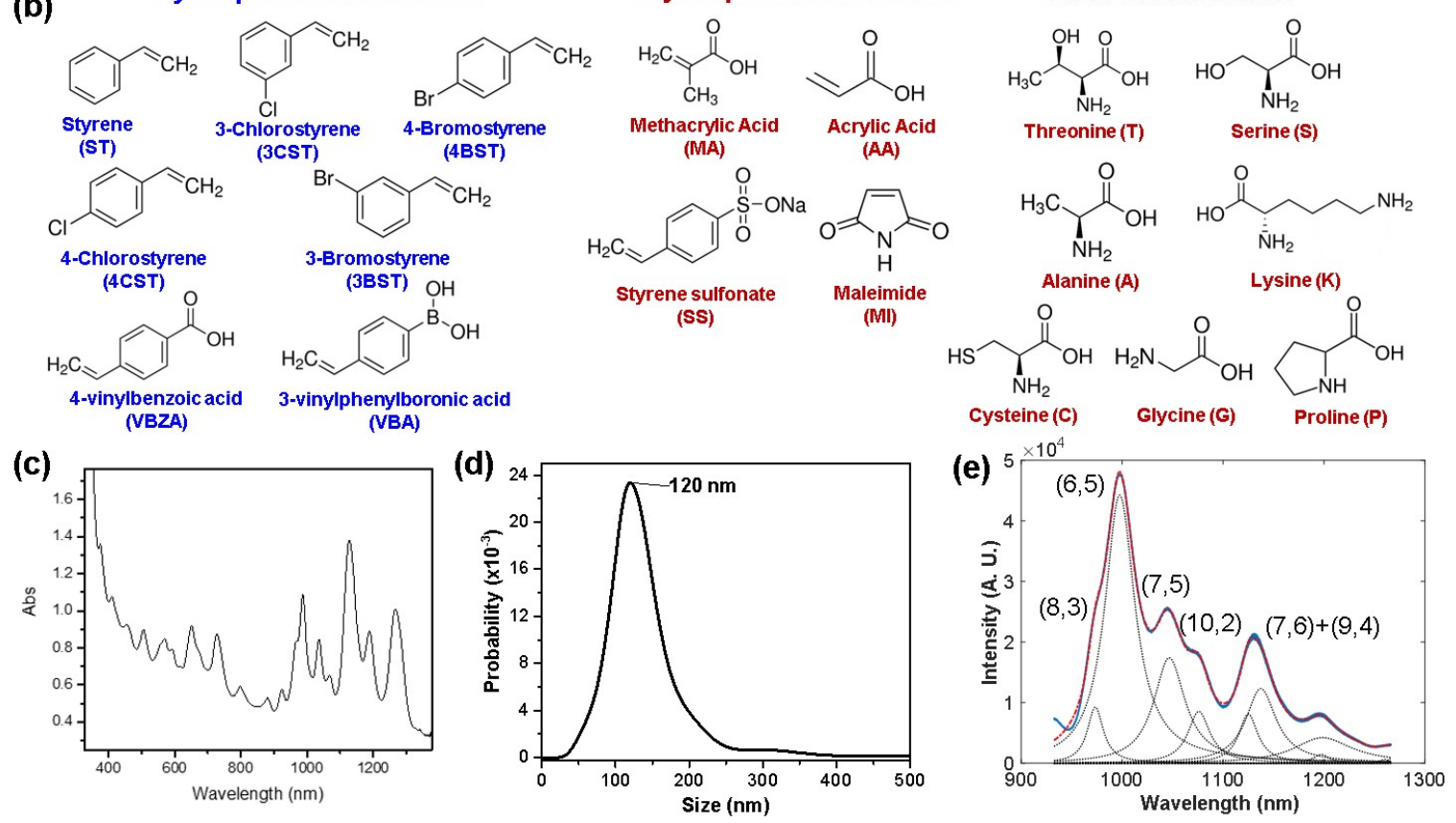

Figure 1: Analyte molecules, polymer components and nanotube colloidal solution characterizations. (a), Structures of 5 small therapeutics screened as the initial targets. (b), Structures of hydrophobic and hydrophilic monomers, together with post-polymerization modifications using amino acids. (c), The UV-vis-nIR absorption spectrum of nanotubes with MA-ST-90 polymer corona, showing distinct and sharp peaks of $E_{11}$ and $E_{22}$ transitions, indicating the successful isolation of individual nanotubes. (d), Dark-field scattering microscopy is used to analyze individual particle size based on their Brownian motions, showing a single population of nanotubes with MA-ST-90 corona, with an average hydrodynamic size about $60 \mathrm{~nm}$. (e), The near infrared emission spectrum of nanotubes under $785 \mathrm{~nm}$ excitation is deconvoluted to quantify the contribution of each chirality. Changes in emission spectra will be used to quantify the interaction between analytes and corona phases. 


\section{Results and Discussion}

\section{Corona phase recognition for Vardenafil}

To build artificial molecular recognition sites, we have designed a library of 24 amphiphilic polymers as the corona, aiming for small therapeutic recognition (Fig. 1a and b). Polymers are composed of alternating hydrophobic and hydrophilic segments. The hydrophobic part uses aromatic monomers like styrene and its derivatives for their strong $\pi-\pi$ stacking on nanotube surface, serving as anchors for polymer adsorption; the hydrophilic part composes functional monomers such as methacrylic acid, styrene sulfonate and maleimide, responsible for supramolecular interactions with analytes. Post-polymerization modifications with amino acids are introduced to increase the structural and conformational diversity (Fig. 1b). These polymers are synthesized using reversible addition-fragmentation chain-transfer (RAFT) polymerization for its narrow polydispersity, with the initiator azobisisobutyronitrile (AIBN). The abbreviation of polymer names are assigned using their monomer's acronym (Fig. 1b), followed by the composition percentage of first monomer (for example, polymer MA-ST-90 is made of methacrylic acid and styrene at a 90:10 ratio). (See Supporting Info. for polymer characterizations.) HiPCO nanotubes are used in order to screen various chirality of nanotubes. Polymer corona on nanotubes was achieved by sonicating nanotubes in aqueous polymer solutions, where amphiphilic polymers fold on nanotube surfaces and stabilize individual nanotubes. Residual nanotube bundles are discarded by ultracentrifugation (105000 rfc for $4 \mathrm{~h}$ ). The resulting colloidal solution is characterized by UV-vis-nIR absorption spectroscopy and dark-field scattering microscope. Spectra in Fig. 1c and $\mathrm{d}$ are of corona phase MA-ST-90, showing that there is one main population of isolated nanoparticles, with a hydrodynamic size around $60 \mathrm{~nm}$.

By examining the interactions between the resulting corona phases and small therapeutics, we have identified one corona that specifically recognizes Vardenafil. More specifically, therapeutic molecules (at $2 \mu \mathrm{M}$ ) are introduced into SWNT colloidal solution and the corre- 
sponding nIR spectral change is quantified. The spectral response of each nanotube chirality is examined by deconvoluting the emission spectra (Fig. 1e). For nanotubes of (8,3) and $(6,5)$ chirality (whose peaks are very close in emission spectra, hence analyzed together), most of corona phases have shown little intensity change (Fig. 2a), less than 15\%, possibly due to concentration variations among triplicates. For polymer coronas that have shown strong spectral change, some have responded to multiple analytes, suggesting a nonspecific interaction. For example, Vardenafil, Bupropion and Sumatriptan all have caused more than $20 \%$ intensity increase in MA-ST-G-75 wrapped SWNTs. In contrast, MA-ST-90, made of methacrylic acid and styrene, has shown $\approx 26 \%$ intensity reduction, only in response to Vardenafil. In addition, among all the coronas, Vardenafil has only caused strong emission reduction in this corona phase. Moreover, considering that almost all intensity changes in methacrylic acid polymers are turn-on responses, it is especially unique to have a strong turnoff response in corona MA-ST-90. All these features suggest that it is a potential specific recognition of Vardenafil by this polymer corona phase.

By expanding the analyte library to 22 small molecules of different chemical structures, we have confirmed the recognition specificity. The MA-ST-90 templated nanotubes did not show strong interactions with any other analyte (Fig. 2b). We did not observe any correlation between analyte hydrophobicity and the emission modulation (Fig. 2c). This result demonstrates that the recognition by corona MA-ST-90 is not caused by nonspecific absorption, but by a special interaction with Vardenafil.

Besides the specificity, we have also confirmed that the emission reduction of nanotubes is not a result of particle aggregation. The hypothesis is that if Vardenafil non-specifically disrupts the electrostatic repulsion between particles, it will cause bundling of nanotubes, which will also reduce the emission intensity. Using a dark-field scattering microscope to track individual nanoparticle's Brownian motion, we have quantified the hydrodynamic sizes of nanoparticles. As shown in Fig. 2d, the distribution of nanotubes' hydrodynamic sizes is consistent with or without Vardenafil presented $(66 \mathrm{~nm}$ vs. $68 \mathrm{~nm})$, falsifying the bundling 

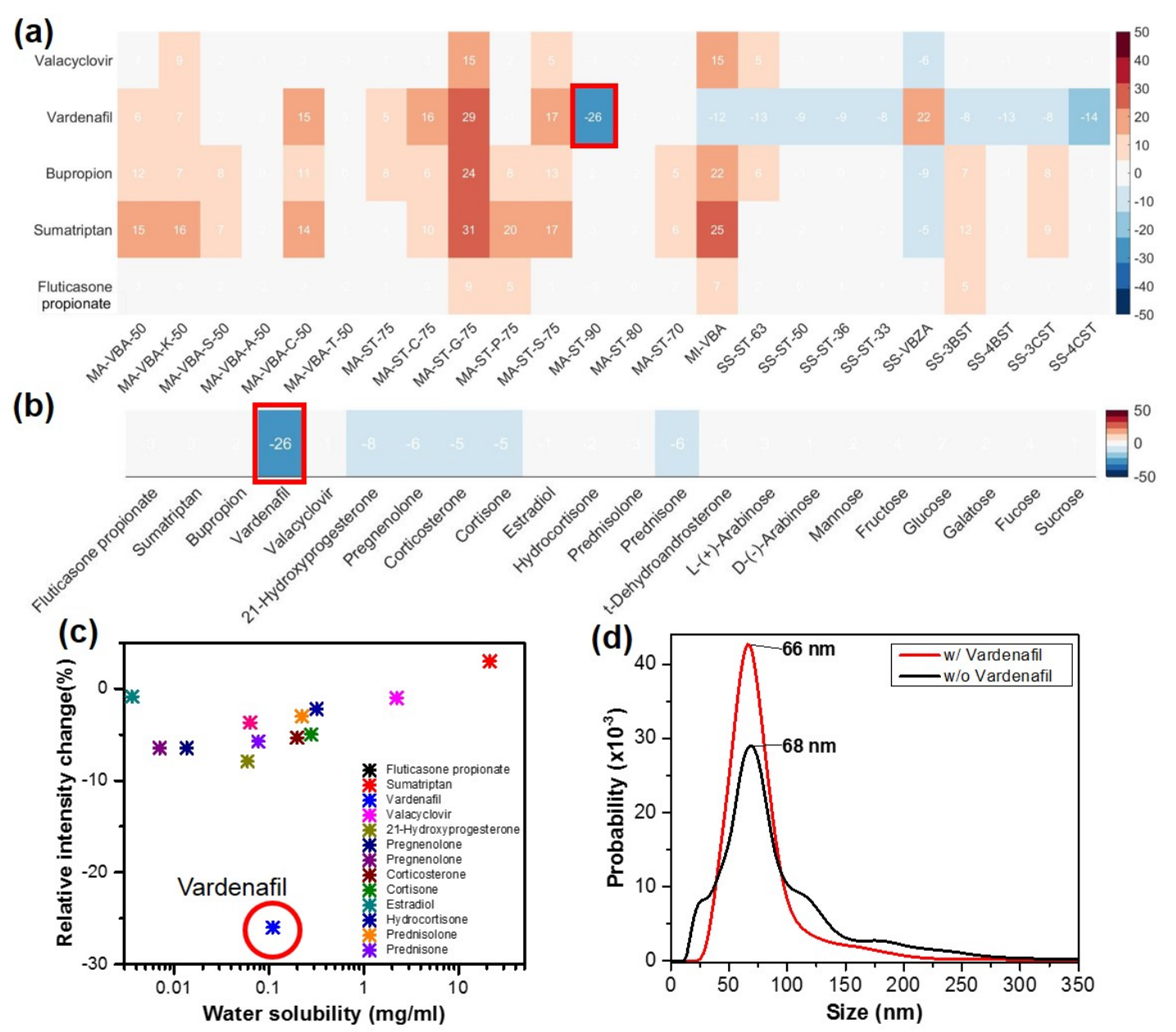

Figure 2: Fluorescence intensity change of nanotube coronas in response to drug analytes. (a), A library of amphiphilic polymers was synthesized to form coronas on nanotubes. The near infrared emission change of these nanotubes is examined when small therapeutics are introduced (at $2 \mu \mathrm{M}$ ). With the poly(methacrylic acid-co-styrene) (MA-ST-90) corona, nanotubes of $(8,3)$ and $(6,5)$ chirality exhibit strong quenching in response to Vardenafil, in contrast to other corona phases that did not show any specific change. (b), When the analyte library is expanded to 22 small molecules, Vardenafil remains as the only analyte that causes strong nIR emission change in MA-ST-90 corona. (c), No correlation was observed between the emission intensity change and the hydrophobicity of analyte, indicating that the quenching is not due to hydrophobic absorption. (d), The hydrodynamic size of nanotubes with corona MA-ST-90 was not influenced by Vardenafil binding ( $66 \mathrm{~nm}$ vs. $68 \mathrm{~nm}$ ), falsifying the hypothesis that the quenching is a result of nonspecific particle aggregation cause by Vardenafil.

hypothesis.

These investigations demonstrate that the recognition of Vardenafil by MA-ST-90 corona can't be attributed to physical properties of analyte or nonspecific interactions among par- 
ticles, supporting the specific recognition mechanism. The following section examines the nature of this recognition interaction.

\section{Recognition mechanism}

\section{Structural properties of poly(methacrylic acid-co-styrene)}

The recognition interaction is very sensitive to the hydrophobic-hydrophilic composition of the corona phase. Polymer MA-ST-75, made of the same monomers as in MA-ST-90, did not show a strong interaction with Vardenafil in the initial screening (Fig. 2a). MA-ST90 has a hydrophilic:hydrophobic ratio of 9:1, in comparison of 7.5:1 in MA-ST-75. For $(6,5)$ and $(8,3)$ nanotubes, when different concentrations of Vardenafil were introduced, MAST-90 exhibits a $15 \%$ to $35 \%$ intensity reduction as the analyte concentration increases (Fig. 3a and c). In contrast, MA-ST-75, shows a much smaller intensity modulation, and was not concentration-dependent (Fig. 3b and c). Moreover, for MA-ST-90, the emission wavelength redshifts, reaching about $4 \mathrm{~nm}$ at $5 \mu \mathrm{M}$, whereas the MA-ST-75 emission did not change (Fig. 3f). Besides the emission spectra, we have also collected the excitation-emission spectra to examine the resonant $E_{22}$ emissions. Similar intensity and wavelength changes are observed for MA-ST-90, but not for MA-ST-75 (Fig. 3d, 3e and Supporting Info.) Results from both spectra demonstrate that the higher hydrophilic:hydrophobic ratio is essential for the recognition. Because a higher MA:ST ratio is associated with less hydrophobic "anchors", such a necessity on the composition ratio indicates that the recognition requires the corona to have a certain degree of flexibility. So that when Vardenafil approaches, the polymer corona can adapt its configuration to have a strong interaction with Vardenafil.

Changing polymer length can also modulate the response of corona phases to analyte. Very interestingly, when poly(methacrylic acid-co-styrene) lengthens from $10 \mathrm{kDa}$ to $15 \mathrm{kDa}$, the emission intensity change caused by Vardenafil binding gradually changes from a turn-off to a turn-on response, as shown in Fig. 4a and c. ( $M_{w}$, see Supporting info. for gel permeation chromatography results.) The trend is also consistent at different analyte concentrations. 

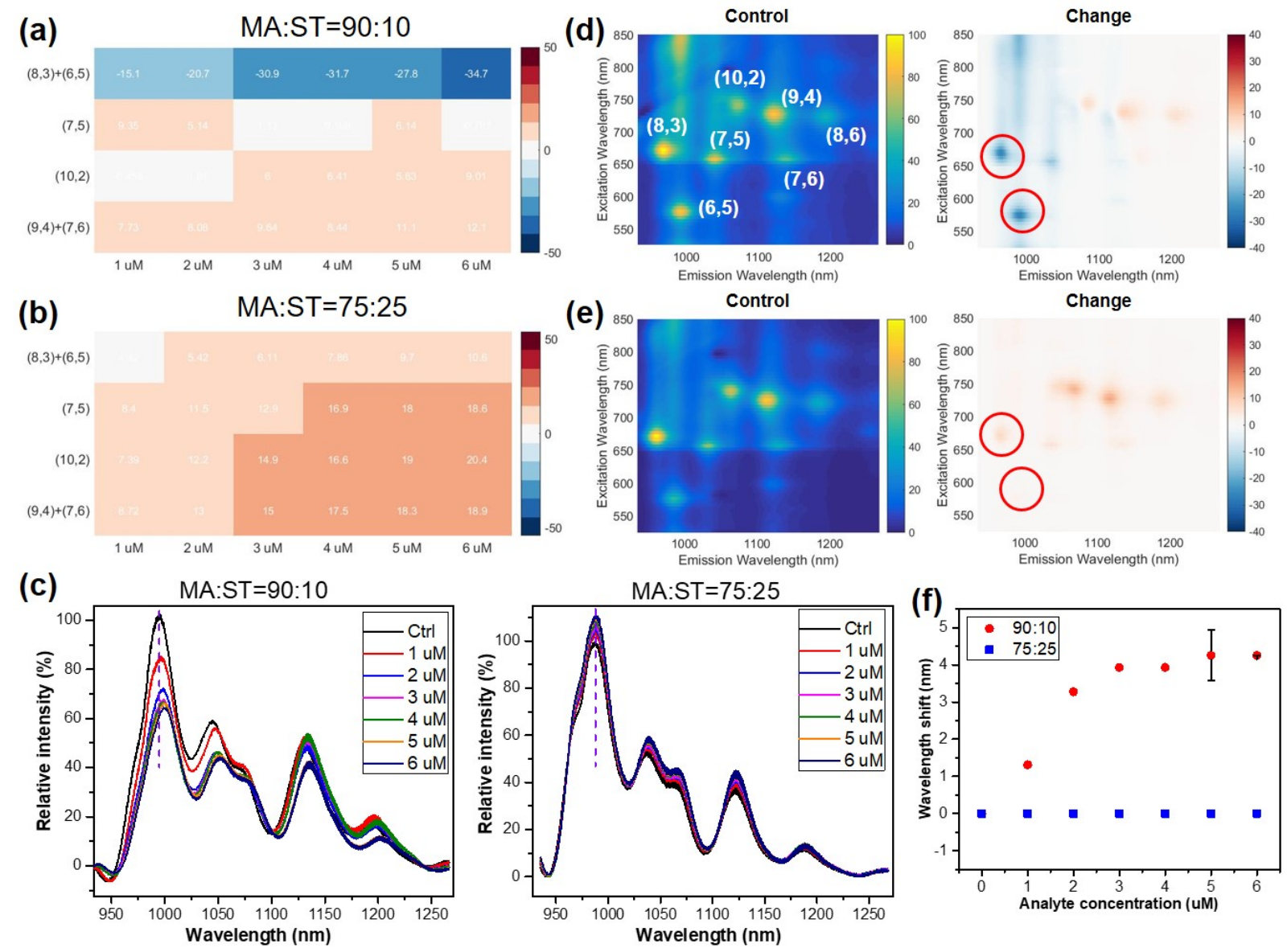

Figure 3: The hydrophobic-hydrophilic ratio of polymer corona influences the recognition interaction. (a), Nanotubes of $(6,5)$ or $(8,3)$ chirality, with the MA-ST-90 corona, show a gradual emission reduction as Vardenafil concentration increases. (b), Emission intensity modulation in MA-ST-75, which has a lower hydrophilic:hydrophobic ratio, is much smaller and does not change with the analyte concentration. (c), Emission spectra of the two corona phases when Vardenafil of $1-6 \mu \mathrm{M}$ was introduced, showing a strong intensity change and wavelength shift in MA-ST-90, but not in MA-ST-75. (d), The excitation-emission spectrum of MA-ST-90 corona phase and its change upon the introduction of Vardenafil. The spectrum presents resonant emission peaks, each corresponding to a nanotube chirality, labeled with a pair of numbers. A strong reduction, similar to the ones in emission spectra, is observed for $(6,5)$ and $(8,3)$ nanotubes. (See Supporting Info. for quantified wavelength shift.) (e), In contrast, the excitation-emission spectrum of MA-ST-75 did not show major intensity or wavelength shift. (f), The emission peak of MA-ST-90 corona phase redshifts as a result of the dielectric constant change caused by Vardenafil binding. The magnitude increases with analyte's concentration. Color bar units in heat maps are all in percentage.

In addition, the dissociation constants $\left(K_{D}\right)$ of recognition interaction can be extracted by calibrating the intensity change at different analyte concentrations. The $K_{D}$ are on the order 
of micro molar, with the lowest being $0.4 \mu \mathrm{M}$ (Fig. $4 \mathrm{~d}$ ), comparable to antibody's affinity. On the other hand, the wavelength shifts, are similar among all polymer lengths: all towards lower energy by $2-4 \mathrm{~nm}$ (Fig. 4b). The consistent wavelength change suggest that the polymer length does not influence the recognition capacity: Vardenafil binds specifically to nanotube coronas and results in a consistent increase in dielectric constant.

The different intensity changes in coronas result from different surface coverage of nanotubes. We used two characterizations to quantify nanotube surface coverage: the solvatochromic shifts in emissions and the absorption of dye molecules. The solvatochromic shifts of resonant emissions are extracted from excitation-emission spectra. Based on a semiemperical estimation, ${ }^{13,17}$ when compared with pristine nanotubes in the air, the transition energy shift in corona nanotubes $\left(\Delta E_{11}\right)$ is correlated to the effective dielectric constant $\left(\varepsilon_{e f f}\right)$, which is a linear combination of dielectric constants of water and polymer (Eqn (1$3)$ ). The ratio of this linear combination $(\alpha)$ is decided by the surface coverage of nanotubes (see Supporting Info for details). As shown in Fig. 4e, compared to the $10 \mathrm{kDa}$ polymer, the $14 \mathrm{kDa}$ polymer has a smaller slope in solvatochromic plot, correlating to a smaller dielectric constant and larger surface coverage $(59.5 \%$, in comparison to $34.2 \%)$. When comparing coronas of variable lengths, there is a clear increase of nanotube surface coverage, from less than $40 \%$ to $60 \%$, as the polymer length extends to $12 \mathrm{kDa}$ (Fig. $4 \mathrm{f}$ ).

$$
\begin{aligned}
\left(E_{11}\right)^{2} \Delta E_{11}= & -L k\left[\frac{2(\varepsilon-1)}{2 \varepsilon+1}-\frac{2\left(\eta^{2}-1\right)}{2 \eta^{2}+1}\right]\left(\frac{1}{d^{4}}\right)=\frac{c}{d^{4}} \\
\frac{c}{c_{\text {ref }}}= & \frac{\frac{\varepsilon_{e f f}-1}{2 \varepsilon_{\text {eff }}+1}-\frac{\eta^{2}-1}{2 \eta^{2}+1}}{\frac{\varepsilon_{\text {ref }}-1}{2 \varepsilon_{\text {ref }}+1}-\frac{\eta_{\text {ref }}^{2}-1}{2 \eta_{\text {ref }}^{2}+1}} \\
\varepsilon_{\text {eff }}= & \alpha \varepsilon_{\text {polymer }}+(1-\alpha) \varepsilon_{\text {water }}
\end{aligned}
$$

where $E_{11}$ is the transition energy with the corona, $E_{11}^{a i r}$ is the transition energy in air, $L$ 

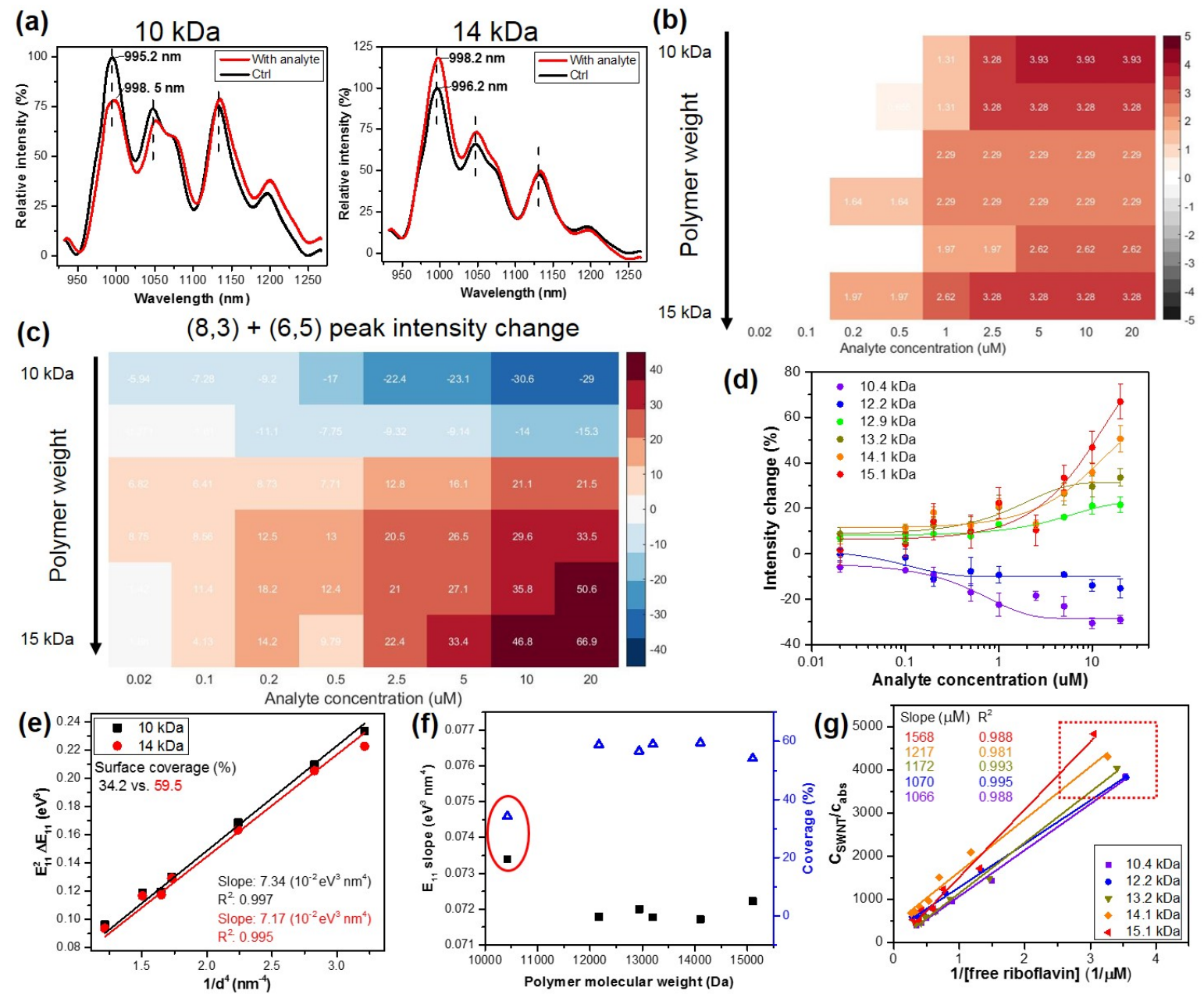

Figure 4: Polymer length modulates the corona phase response to the analyte. (a), Coronas made of poly(methacrylic acid-co-styrene) of different lengths exhibit opposite emission intensity change in response to $2 \mu \mathrm{M}$ Vardenafil. Both show redshift in emission wavelength. (b), Corona phases made of polymers of different lengths, from $10 \mathrm{kDa}$ to $15 \mathrm{kDa}$, all show similar redshift in nanotubes' nIR emissions. (c), As the polymer lengthens from 10 to $15 \mathrm{kDa}$, the nIR emission of $(6,5)+(8,3)$ nanotubes gradually changes from turn-off response to turn-on response, consistent over different Vardenafil concentrations. (d), The concentration-dependent intensity changes are calibrated to calculate the dissociation constants, with the lowest $K_{D}$ being $0.4 \mu \mathrm{M}$, comparable to the affinity of antibodies, indicating a strong recognition interaction between nanotube corona and Vardenafil. (e), Solvatochromic plots characterize the polymer coverage on nanotube surface (see Eqn (1)). The surface coverage by the $10 \mathrm{kDa}$ polymer is $34.2 \%$ (black squares), much smaller than that of the $14 \mathrm{kDa}$ polymer, at $59.5 \%$ (red circles). (f), Based on the slopes of solvatochromic plots (black solid squares), polymers that are longer than $12 \mathrm{kDa}$ have a greater coverage on nanotubes (about $60 \%$ ). (g) When mixed with dyes, coronas of longer polymer length lead to higher concentration of free riboflavin, indicating a smaller exposed nanotube surface, thus reaching a similar conclusion that longer MA-ST-90 polymers have higher surface coverage 
Figure 4: (Continued) on nanotubes. Red box highlights the comparison at the lowest dye concentration. Colorbars for heat maps are all in the unit of percentage.

is a fluctuation factor, $k$ is a scaling constant of the SWNT polarizability, $d$ is nanotube's diameter, $c$ is the slope in the linear fitting in Eqn (1), $\varepsilon$ is the dielectric constant, $\eta$ is the reflective index, and $\alpha$ is the coverage percentage of nanotube surface.

We have reached similar conclusions about the surface coverage by quantifying the dye absorption to the exposed surface of nanotubes. More specifically, when riboflavin was introduced into nanotube colloidal solutions, they absorb onto nanotube surface due to hydrophobic interaction and their emission is quenched. The absorption amount is proportional to the exposed nanotube surface area, and can be quantified by calibrating the quenching. As presented in Fig. 4g, as the polymer lengthens, the free riboflavin concentration increases (in the red box), indicating a reduction of nanotube surface exposure. The linear plot is governed by the equation: $\frac{C_{\text {swnt }}}{c_{\text {abs }}}=\frac{K_{\text {swnt-dye }}}{q} \cdot \frac{1}{c_{\text {free }}}$, where $C_{\text {swnt }}$ is the $\mathrm{C}$ atomic concentration of SWNTs; $c_{a b s}$ is the concentration of dyes absorbed; $c_{f r e e}$ is the free riboflavin concentration, $K_{\text {swnt-dye }}$ is the dissociation constant, and $q$ is the number of riboflavin molecules absorbed per $\mathrm{C}$ atom (see Supporting Info for detailed derivation). Because it is a physical absorption process, it is reasonable to assume that $K_{\text {swnt-dye }}$ is a constant among different coronas, therefore, the slope reflects the number of riboflavin absorbed. As the polymer lengthens, the slope increases from 1066 to $1568 \mu \mathrm{M}$ (Fig. 4g), demonstrating that the nanotubes have less hydrophobic surface exposed, similar to the results from solvatochromic shifts. Both characterizations conclude that shorter polymers pack less densely on nanotubes, thus allowing Vardenafil to interfere SWNTs' bandgap and causing quenching. When the surface coverage is high, the interference effect is not efficient, and fluorescence intensity change is caused by the corona configuration modulation when interacting with Vardenafil.

Both methacrylic acid and styrene are essential for the corona phase to recognize Vardenafil. Two control polymers poly(acrylic acid-co-styrene (AA-ST) and poly(methacrylic acidco-vinylphenylboronic acid) (MA-VBA) were synthesized by replacing either the methacrylic 

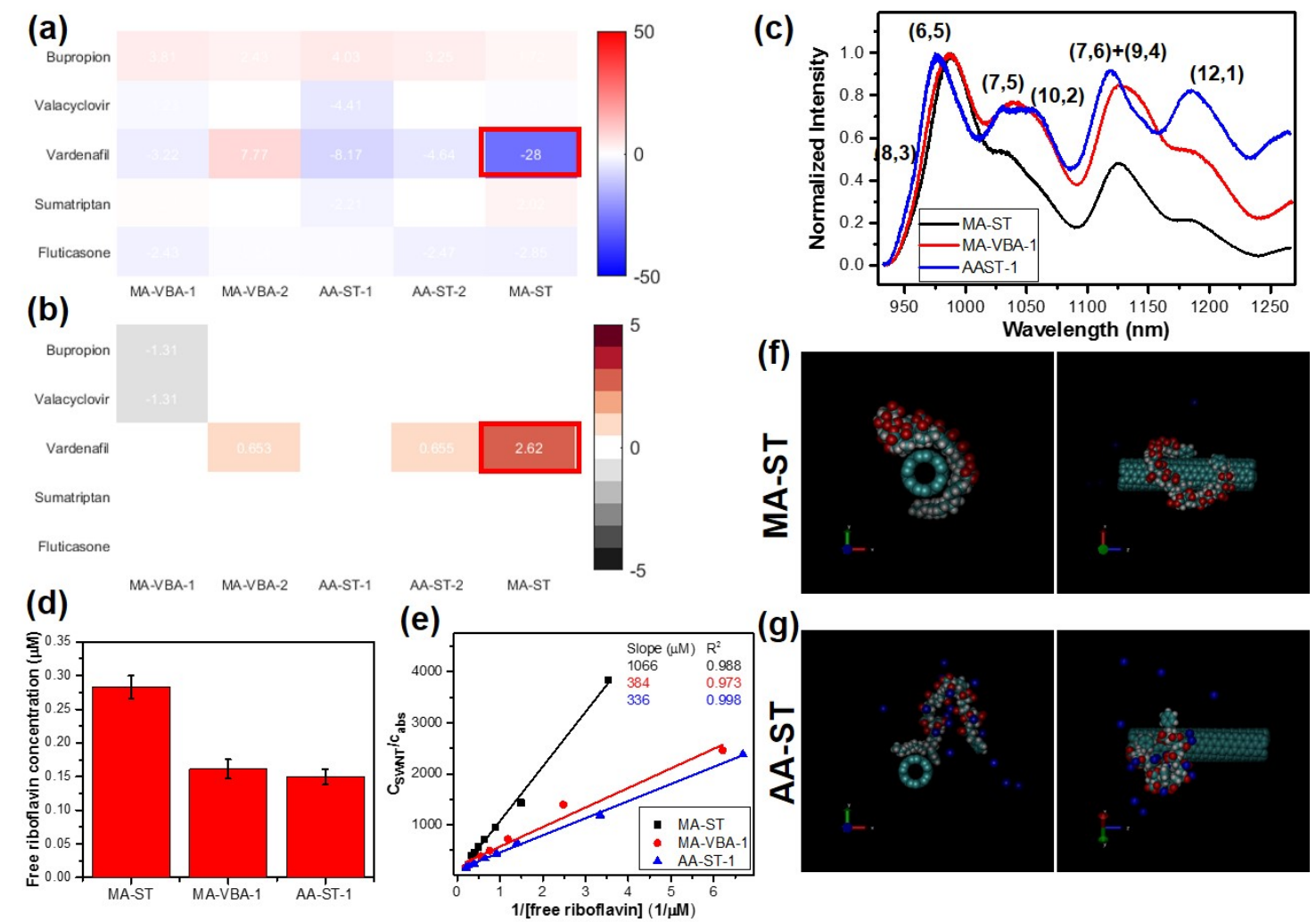

Figure 5: Monomer methacrylic acid and styrene are essential for the recognition of Vardenafil. (a) and (b), When methacrylic acid is replaced with acrylic acid, or when styrene is replaced with 3-vinylphenolboronic acid, the resulting corona phase AA-ST an MA-VBA do not show a strong interaction with Vardenafil. Neither the intensity (a), nor the wavelength (b) shows a substantial change. (c), Comparison of the emission spectra of the three corona phases demonstrates that MA-ST has a preference for small diameter nanotubes, while the other two do not. (d), When riboflavin was introduced into the nanotube solution with MA-ST corona, more than half of dye molecules are free in solution. Whereas, less than $30 \%$ are free when in the solution with AA-ST or MA-VBA coronas, indicating MA-ST has a much higher surface coverage on nanotubes, consistent with the conclusion from linear slope values in (e). (f) and (g), Molecular dynamic simulations of polymer segments show their corona configurations: MA-ST forms a binding pocket on nanotubes, whereas AA-ST does not. Left: the cross section; right: the side view.

acid or the styrene. Both polymers are made of 90:10 hydrophilic-hydrophobic ratio like in MA-ST, with polymer lengths around $10 \mathrm{kDa}$ (AA-ST-1 and MA-VBA-1) or $15 \mathrm{kDa}$ (AA-ST2 and MA-VBA-2). None of the control polymers was able to generate a corona phase that recognizes Vardenafil, supported by the lack nIR spectral change (Fig. 5a and b). When analyzing the difference among coronas, we found that MA-ST corona has a strong $(8,3)+(6,5)$ 
emission, in contrast to AA-ST and MA-VBA corona phases that have similar intensities among different chirality. Considering that the $(8,3)+(6,5)$ nanotubes have the strongest interaction with analyte (Fig. $2 \mathrm{a}$ and Fig.4c), the preference of small diameter nanotubes by MA-ST polymers is critical for their recognition of Vardenafil. On the other hand, the surface coverage of nanotubes is much higher with MA-ST corona when compared with AAST and MA-VBA. As shown in Fig. $5 \mathrm{~d}$, about $0.27 \mu \mathrm{M}$ out of $0.5 \mu \mathrm{M}$ of riboflavin is free in MA-ST nanotube solution, compared to more than $70 \%$ absorption in control polymers. The trend is consistent when multiple concentrations of riboflavin was used (Fig. 5e), where the slope of linear fitting for MA-ST corona is 3 times higher than those of MA-VBA and AA-ST, suggesting that MA-ST has a much higher surface coverage.

Molecular dynamic simulation shows a "binding pocket" configuration in MA-ST corona on nanotubes, but not in AA-ST. The configurations were simulated in the presence of wa-

ter for $50 \mathrm{~ns}$ to reach equilibrium. (A $2200 \mathrm{Da}$ segment of polymers and one unit length of $(6,5)$ SWNT are used in simulations to save the computational time.) In reaching the equilibrium, the MA-ST polymer gradually wraps around SWNT, reducing the solvent access surface from 15.03 to $14.03 \mathrm{~nm}^{2}$. The resulting configuration forms a "binding pocket" enclosed by nanotube and polymer surfaces (Fig. 5f). In contrast, the folding of AA-ST on nanotubes reduced the solvent access surface from 15.03 to $14.71 \mathrm{~nm}^{2}$, a smaller reduction, and did not form a stable binding configuration. Both the experimental and simulation results demonstrate that the specific recognition of Vardenafil results from the overall structure of MA-ST and its corona configuration on nanotubes.

\section{MA-ST corona phase in mimicking PDE5 for Vardenafil recognition}

In comparison with natural recognition site PDE5, MA-ST corona phase selectively binds to two inhibitors, Vardenafil and Sildenafil, not to Tadalafil and the substrate cGMP. As shown in Fig. 6a, the biological role of Vardenafil is to interrupt the enzymatic function of PDE5 in converting cyclic guanosine monophosphate (cGMP) to guanosine monophosphate (GMP). 
(a)
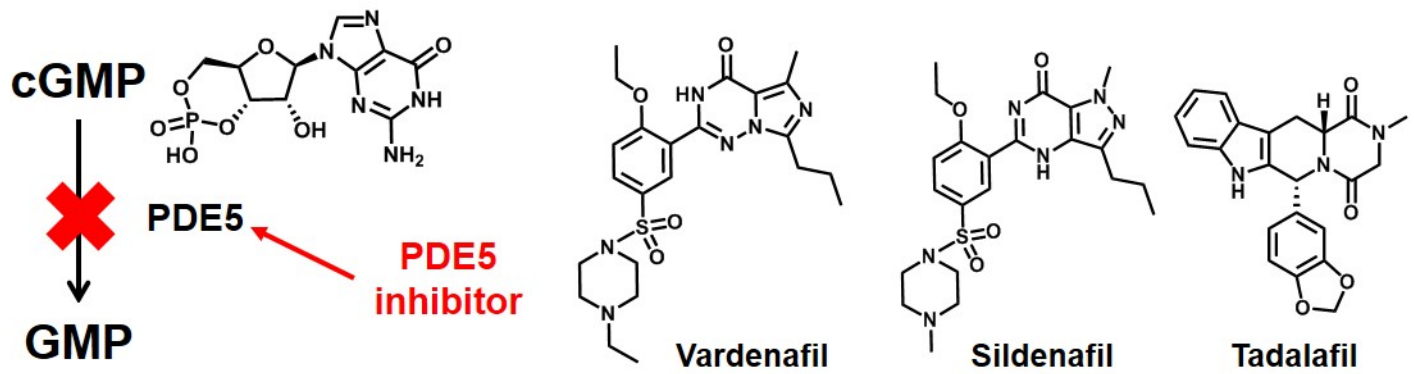

Tadalafil

(b)

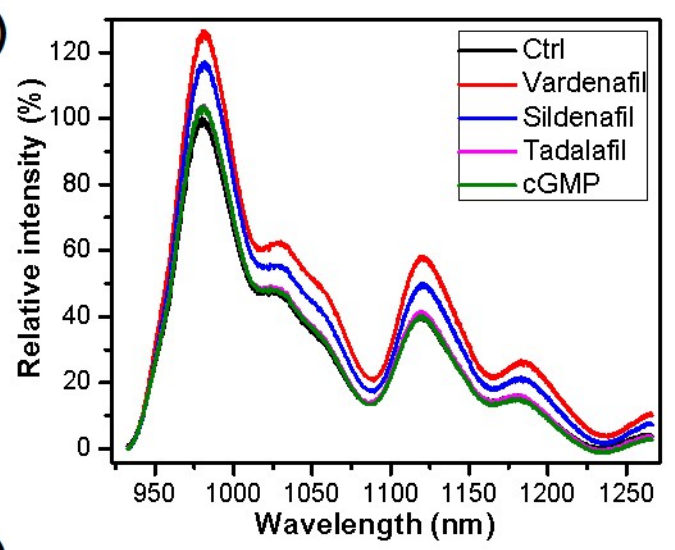

(d)

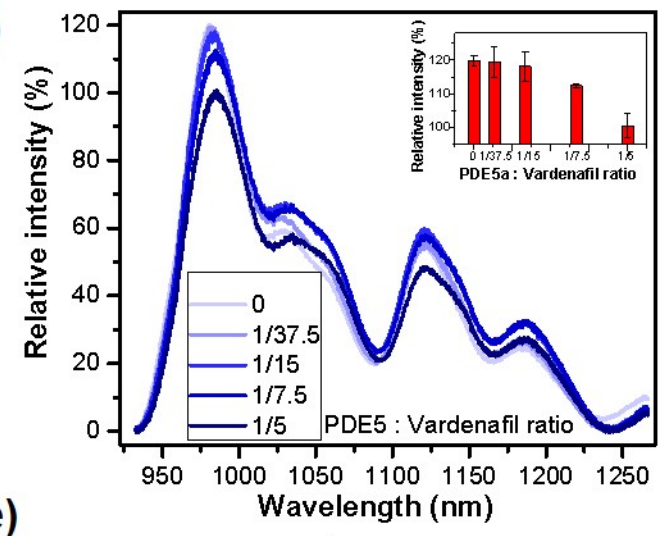

(c)
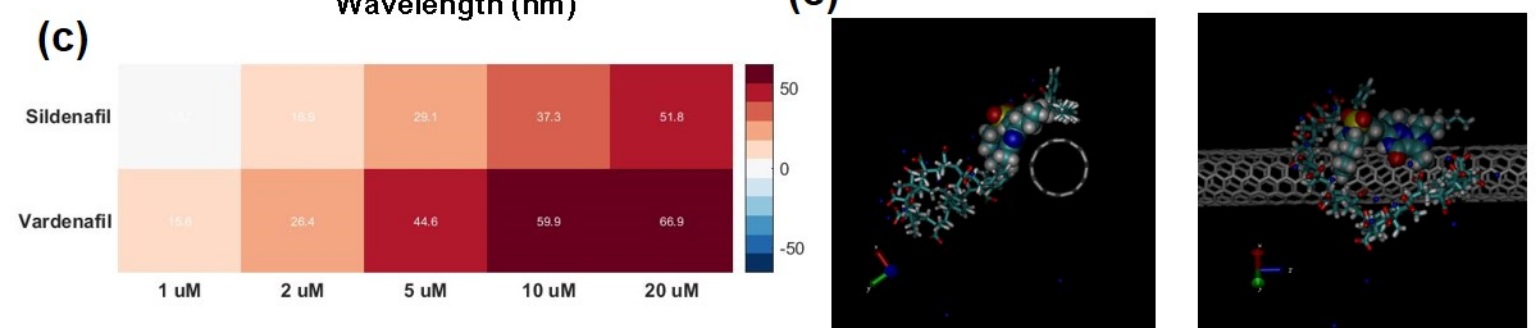

Figure 6: MA-ST corona phase in mimicking PDE5 for Vardenafil recognition. (a), The biological role of Vardenafil is to bind PDE5 and interrupt its function in converting cGMP to GMP. Sildenafil and Tadalafil are two other PDE5 inhibitors. (b), The nIR spectral responses of MA-ST corona phase to PDE5 substrate (cGMP) and inhibitors. Only Vardenafil and Sildenafil have shown strong interactions with MA-ST coronas. (c), The emission intensity modulation increases as the concentrations of Sildenafil and Vardenafil increase. The binding affinity is smaller for Sildenafil. (d), PDE5a enzyme interferes with the interaction between Vardenafil and the corona phase, gradually eliminating it as the enzyme concentration increases. The inset is the relative intensity change of the $(6,5)$ peak at different PDE5a concentrations. (e), Molecular dynamic simulation shows the configuration of Vardenafil docked in the binding pocket of MA-ST corona.

Besides Vardenafil, Sildenafil and Tadalafil are PED5 inhibitors too. Only Vardenafil and Sildenafil present strong interactions with MA-ST corona phase $(15 \mathrm{kDa})$, whereas cGMP and Tadalafil did not (Fig. 6b). The observation can be explained by the different interactions between these molecules and PDE5. Sildenafil has a similar chemical structure as Vardenafil 
and interacts with same residues in the H-loop of the catalytic site in PDE5. ${ }^{18}$ Tadalafil, on the other hand, has a very different structure and interacts with the M-loop of the catalytic site. ${ }^{18,19}$ As the substrate, cGMP binds to both the catalytic site and the allosteric site, whereas the inhibitors do not, ${ }^{20-22}$ indicating a different binding mechanism in the substrate. The result suggests that our corona phase recognition shares similar interaction characters as in the H-loop in PDE5. A further look at various analyte concentrations, Sildenafil consistently causes a smaller intensity change than Vardenafil (Fig.6c), demonstrating a smaller binding affinity to the corona phase. This is a similar trend as in their interactions with PDE5, where Vardenafil has a 10- to 40-fold tighter binding. ${ }^{18}$

In the presence of enzyme interference, the interaction between MA-ST corona and Vardenafil is outcompeted. When Vardenafil was introduced into a nanotube solution with PDE5a (a subset of PDE5), its binding with the corona phase gradually weakens as the PDE5a concentration increases (Fig.6d). When the ratio between PDE5a and Vardenafil reaches $1 / 5$, no nIR spectral change is presented as PDE5a outcompetes the corona phase. The result reveals that PDE5a has a higher binding affinity than the corona phase. The disruption of the recognition in the presence of a competitor of higher binding affinity further proves that the MA-ST corona phase has a specific interaction with Vardenafil.

Molecular dynamic simulation depicts that Vardenafil docks into the "binding pocket" of corona phase as it is being recognized (Fig. 6e). During this recognition process, nanotubes have a larger surface coverage (reducing the solvent access surface from 14.03 to $13.23 \mathrm{~nm}^{2}$ ). The corona has a slightly different configuration compared to before binding, supporting our experimental observation that some flexibility is needed in the corona phase to facilitate the recognition. Similar configurational change also happend when Vardenafil binds to the H-loop in PDE5, ${ }^{23}$ further demonstrating the power of our recognition site. 


\section{Summary}

In summary, we have generated a synthetic mimic of PDE5 to bind its inhibitor Vardenafil, using single-walled carbon nanotube templated corona phase recognition. Among a library of amphiphilic polymers, the corona phase made of poly(methacrylic acid-co-styrene) stands out for its specific binding to Vardenafil. The recognition is not related to physical properties of the analyte or the corona. The interaction is proven to be highly sensitive to the 3D configuration of the corona phase: the change of polymer composition sabotages the recognition; and the change of polymer length modulates the spectral response. In comparison with the natural binding enzyme, the MA-ST corona phase is more selective, only recognizing Vardenafil and a structure very similar to it, but not the other inhibitor or substrate, indicating that the corona shares similar properties as the H-loop of PDE5. The corona phase has a smaller binding affinity than the enzyme, as the interaction is disrupted in the presence of PDE5a. Molecular dynamic simulation demonstrates the interaction origins from a "binding pocket" in MA-ST corona on nanotubes, which is responsible for the specific recognition of Vardenafil. The unique artificial recognition site for inhibitors will open up possibilities for new pharmaceutical and biological applications.

\section{Acknowledgments}

This work is supported by GlaxoSmithKline plc.

\section{References}

(1) Weber, C. A.; Mehta, P. J.; Ardito, M.; Moise, L.; Martin, B.; De Groot, A. S. Advanced Drug Delivery Reviews 2009, 61, 965-976.

(2) McEnaney, P. J.; Fitzgerald, K. J.; Zhang, A. X.; Douglass, E. F.; Shan, W.; Balog, A.; Kolesnikova, M. D.; Spiegel, D. A. J. Am. Chem. Soc. 2014, 136, 18034-18043. 
(3) Chames, P.; Van Regenmortel, M.; Weiss, E.; Baty, D. Br J Pharmacol 2009, 15\%, $220-233$.

(4) Beck, A.; Wurch, T.; Bailly, C.; Corvaia, N. Nature Reviews Immunology 2010, 10, 345-352.

(5) Han, Y.; Meng, Z.; Ma, Y.-X.; Chen, C.-F. Acc. Chem. Res. 2014, 47, 2026-2040.

(6) Späth, A.; König, B. Beilstein Journal of Organic Chemistry 2010, 6, 32.

(7) Chen, L.; Wang, X.; Lu, W.; Wu, X.; Li, J. Chemical Society Reviews 2016, 45, 21372211.

(8) Cieplak, M.; Kutner, W. Trends in Biotechnology 2016, 34, 922-941.

(9) Robertson, E. J.; Battigelli, A.; Proulx, C.; Mannige, R. V.; Haxton, T. K.; Yun, L.; Whitelam, S.; Zuckermann, R. N. Acc. Chem. Res. 2016, 49, 379-389.

(10) Kodadek, T.; J. McEnaney, P. Chemical Communications 2016, 52, 6038-6059.

(11) Gotrik, M. R.; Feagin, T. A.; Csordas, A. T.; Nakamoto, M. A.; Soh, H. T. Acc. Chem. Res. 2016, 49, 1903-1910.

(12) Mahon, C. S.; Fulton, D. A. Nature Chemistry 2014, 6, 665-672.

(13) Bisker, G.; Dong, J.; Park, H. D.; Iverson, N. M.; Ahn, J.; Nelson, J. T.; Landry, M. P.; Kruss, S.; Strano, M. S. Nat Commun 2016, 7.

(14) Jin, H.; Heller, D. A.; Kalbacova, M.; Kim, J.-H.; Zhang, J.; Boghossian, A. A.; Maheshri, N.; Strano, M. S. Nature Nanotechnology 2010, 5, 302-309.

(15) Iverson, N. M.; Barone, P. W.; Shandell, M.; Trudel, L. J.; Sen, S.; Sen, F.; Ivanov, V.; Atolia, E.; Farias, E.; McNicholas, T. P.; Reuel, N.; Parry, N. M. A.; Wogan, G. N.; Strano, M. S. Nature Nanotechnology 2013, 8, 873-880. 
(16) Kruss, S.; Landry, M. P.; Vander Ende, E.; Lima, B. M.; Reuel, N. F.; Zhang, J.; Nelson, J.; Mu, B.; Hilmer, A.; Strano, M. J. Am. Chem. Soc. 2014, 136, 713-724.

(17) Choi, J. H.; Strano, M. S. Appl. Phys. Lett. 2007, 90, 223114.

(18) Wang, H.; Ye, M.; Robinson, H.; Francis, S. H.; Ke, H. Mol Pharmacol 2008, 73, $104-110$.

(19) Cahill, K. B.; Quade, J. H.; Carleton, K. L.; Cote, R. H. Journal of Biological Chemistry 2012, 287, 41406-41416.

(20) Rybalkin, S. D.; Rybalkina, I. G.; Shimizu-Albergine, M.; Tang, X.-B.; Beavo, J. A. EMBO J 2003, 22, 469-478.

(21) Okada, D.; Asakawa, S. Biochemistry 2002, 41, 9672-9679.

(22) Turko, I. V.; Francis, S. H.; Corbin, J. D. Biochem J 1998, 329, 505-510.

(23) Wang, H.; Liu, Y.; Huai, Q.; Cai, J.; Zoraghi, R.; Francis, S. H.; Corbin, J. D.; Robinson, H.; Xin, Z.; Lin, G.; Ke, H. Journal of Biological Chemistry 2006, 281, 2146921479 . 